\title{
Adrenal Cortical Carcinoma pT4 TNM Finding v8
}

National Cancer Institute

\section{Source}

National Cancer Institute. Adrenal Cortical Carcinoma pT 4 TNM Finding v8. NCI

Thesaurus. Code C141093.

Tumor of any size that invades adjacent organs (kidney, diaphragm, pancreas, spleen, or liver) or large blood vessels (renal vein or vena cava). (from AJCC 8th Ed.) 